Proc. XII Int. School on Theoretical Physics — Symmetry and Structural Properties of Condensed Matter

\title{
Qubits in Double Quantum Well Structure
}

\author{
P. JakubCzyK, K. Majchrowski and I. Tralle
}

Faculty of Mathematics and Natural Sciences, University of Rzeszów, al. T. Rejtana 16c, 35-959 Rzeszów, Poland

We propose a new approach to producing the qubits in electron ballistic transport in low-dimensional structures such as double quantum wells or double quantum wires. Next, we examine the possibility to produce quantum entanglement in the framework of the Jaynes-Cummings model and shown that at least in principle, the entanglement can be achieved due to series of "revivals" and "collapses" in the population inversion due to the interaction of a quantized single-mode EM-field with a two-level system.

DOI: $10.12693 /$ APhysPolA.132.115

PACS/topics: 72.25.Mk, 74.55.+v, 03.67.Bg

\section{Introduction}

It is well known that by the proper adjusting of the double-quantum wire structure parameters, the system can be designed in such a way as to produce, due to the electron tunnelling across the structure, an assigned transfer of the electron wave function between two wires, while the ballistic electrons move along the wires. It generates the physical qubit which consists of two adjacent quantum wires, while the logical state $|0\rangle$ would be defined by the presence of a single electron in one of them and the logical state $|1\rangle$ by the presence of the electron in an another one [1-3]. We propose here a different approach, in which the qubit could arise as a result of quantum entanglement of the symmetric and antisymmetric states of electrons in the double quantum well (DQW) structure. These states arising due to tunnelling across the structure, while entanglement could be produced and controlled by means of the source of non-classical light.

\section{The model}

We consider the DQW which interacts with classical source of light of the Hamiltonian of the JaynesCummings model (JCM):

$$
\hat{H}=\frac{1}{2} \hbar \omega \sigma_{z}+\hbar \Omega a^{\dagger} a+\hbar\left(a+a^{\dagger}\right)\left(g \sigma_{+}+g^{*} \sigma_{-}\right)
$$

where the part $\frac{1}{2} \hbar \omega \sigma_{z}$ is the two-level Hamiltonian, $\hbar \Omega a^{\dagger} a$ corresponds to free-field Hamiltonian and $\hbar\left(a+a^{\dagger}\right)\left(g \sigma_{+}+g^{*} \sigma_{-}\right)$stands for interaction operator. This model, despite its conceptual simplicity, exhibits interesting and nontrivial features. Namely, it turns out that the Rabi oscillations, which are the common feature of two-level "atom" interacting with classical EM field, are damped independently of the number of photons, a result that is sometimes called "Cummings collapse" (see Ref. [4] for details). However, for longer times, the system exhibits a series of "revivals" and "collapses", which in our opinion, could be used to make the electrons in DQW-structure to be entangled.

As the the DQW structure we choose the material which can be produced on the basis of $S i_{1-x} G e_{x} / G e$ heterostructure [5], where two Si-wells are separated by the $S i_{0.16} G e_{0.84}$-barrier of about $0.35 \mathrm{eV}$ height. The purify of Si and Ge in such structure up to $10^{11} \mathrm{~cm}^{-3}$ or even $10^{10} \mathrm{~cm}^{-3}$ is possible [6].

In Table I we present the results of our simulations for different values of the well $a$ and barrier $b$ widths.

TABLE I

Table I. Results of simulations for different values of the well $a$ and barier $b$ widths.

\begin{tabular}{cccccccc}
\hline \hline & $\begin{array}{c}a \\
{[\mathrm{~nm}]}\end{array}$ & $\begin{array}{c}b \\
{[\mathrm{~nm}]}\end{array}$ & $\begin{array}{c}U_{0} \\
{[\mathrm{eV}]}\end{array}$ & $\begin{array}{c}\epsilon_{1} \\
{[\mathrm{eV}]}\end{array}$ & $\begin{array}{c}\epsilon_{2} \\
{[\mathrm{eV}]}\end{array}$ & $\begin{array}{c}\Delta_{S A S} \\
{[\mathrm{eV}]}\end{array}$ & $\begin{array}{c}\omega \times 10^{13} \\
{[\mathrm{~Hz}]}\end{array}$ \\
\hline 1 & 4 & 2 & 0.355 & 0.1188 & 0.1765 & 0.0577 & 8.7624 \\
2 & 5 & 3 & 0.355 & 0.1039 & 0.1264 & 0.0225 & 3.4169 \\
3 & 5 & 4 & 0.355 & 0.1087 & 0.1209 & 0.0122 & 1.8494 \\
4 & 6 & 4 & 0.355 & 0.0779 & 0.1088 & 0.0309 & 4.6838
\end{tabular}

These simulations was performed for the following values of parameters: $m_{\text {well }}^{*}=0.041 m_{e}, m_{\text {bar }}^{*}=0.0594 m_{e}$ (here $m_{w e l l}^{*}, m_{b a r}^{*}$ are the electron effective masses in the well and in the barrier, respectively and $m_{e}$ is the free electron mass). The $\epsilon_{1}$ and $\epsilon_{2}$ correspond to the energies of two quantum levels arising due to splitting of the ground states caused by the electron tunneling across the barrier; $\Delta_{S A S}$ is the splitting, $U_{0}$ is the height of the barrier, while $\omega$ is nothing else but $\omega=\Delta_{S A S} / \hbar$.

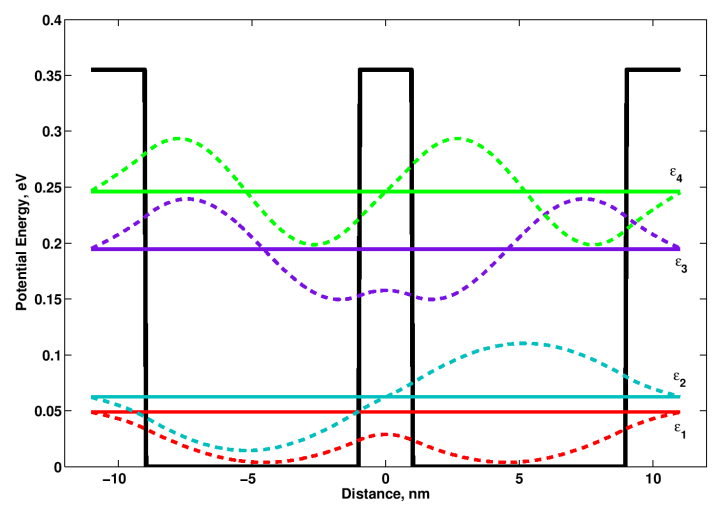

Fig. 1. The energy spectrum of $\mathrm{Si}_{0.16} \mathrm{Ge}_{0.84} / \mathrm{Ge}$ double quantum well and the corresponding stationary wave functions (colored dashed curves). 
In Fig. 1 we depicted the energy spectrum of our DQW-structure which is composed of two quantum wells of $8 \mathrm{~nm}$ width each and the barrier of $2 \mathrm{~nm}$ width.

In this case we have four quantum levels arising due to tunneling across the barrier: $\epsilon_{1} \approx 0.0552 \mathrm{eV}, \epsilon_{2} \approx$ $0.0734 \mathrm{eV}, \epsilon_{3} \approx 0.227 \mathrm{eV}$ and $\epsilon_{4} \approx 0.286 \mathrm{eV}$. The corresponding splittings are equal: $\Delta_{S A S}^{\prime}=0.0182 \mathrm{eV}$ and $\Delta_{S A S}^{\prime \prime}=0.0591 \mathrm{eV}$ and the corresponding frequencies are: $\omega_{S A S 1}=2.7711 \times 10^{13} \mathrm{~Hz}$ and $\omega_{S A S 2}=8.98 \times 10^{13} \mathrm{~Hz}$. The simulations were made by means of Piece-Wise Constant Potential Barriers Tool [7]. In order to carry out these simulations, we determined the widths of the successive layers of which the DQW-structure is composed, the values of electron effective mass within each layer as well as the dependence of potential on the space coordinate. The energy spectrum of DQW-structure and the wave functions were calculated with the use of transfer matrix method [8].

The results of electron concentration calculations in the conduction band of Si, on base of which the DQWstructure could be constructed, are presented in Fig. 2.

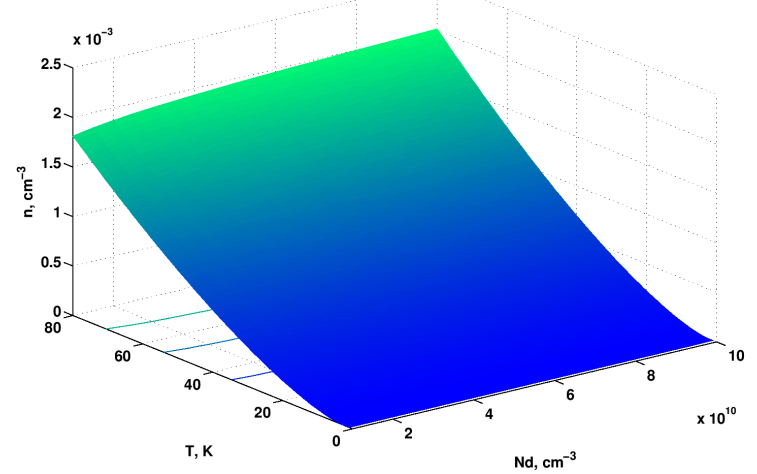

Fig. 2. Electron concentration in Si conduction band vs. donor concentration $N_{d}$ and the temperature.

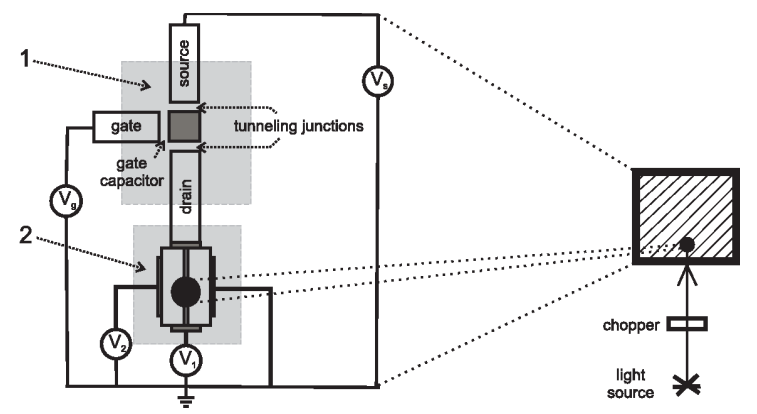

Fig. 3. Layout of possible experiment: $1-\mathrm{SET}, 2-$ DQW (see text).

One can easily notice that the electron concentration in the conduction band is $n \ll 1$ up to the temperature of $80 \mathrm{~K}$. Similar to [2], in order to inject the electrons into DQW-structure one by one, we propose to use the single-electron transistor (SET). The possible layout of the structure is depicted in Fig. 3.

\section{Entanglement}

It is well-known that in order to create entangled states out of product states one needs to have interactions. In our case it is nothing else but the interaction of electron in DQW structure with quantized EM field (photons). This interaction can be described in terms of the JaynesCummings model. Using the basis of dressed states and taking into account that the detunig is not necessarily small, it is convenient to describe the dynamics of the Jaynes-Cummings model in terms of probability of the two-level system to be excited, or in terms of "population inversion" (see $[4,9,10])$ :

$$
\begin{aligned}
& W(t)= \\
& \quad \sum_{n=0}^{\infty} \frac{|\alpha|^{2 n} \mathrm{e}^{-|\alpha|^{2}}}{n !}\left(\frac{\Delta^{2}}{\Omega_{n}^{2}}+\frac{4 g^{2}(n+1)}{\Omega_{n}^{2}} \cos \left(\Omega_{n} t\right),\right.
\end{aligned}
$$

where $W(t)$ is the "population inversion" at $t>0$, $\Omega_{n}=\sqrt{\Delta^{2}+4 g^{2}(n+1)}$ is the Rabi frequency, $\Delta$ is the detuning which in our case is equal to $\Delta=\Delta_{S A S}-\hbar \Omega$; we assume it to be $\Delta \geq 0 ; g$ is the strength of interaction between the two-level system and radiation field, which is also assumed to be greater than zero. Another important parameter characterizing the model is $|\alpha|^{2}$, which is nothing else but the initial number of photons at time $t=0$, before the interaction between two subsystems (two-level "atom" and the photons) starts.

Analysing the dynamics of JMC in terms of probability (2) of the two-level system to be excited, we have shown that one can obtain the entangled states of the form

$$
\left|\Psi_{\text {ent }}\right\rangle \equiv(\beta|0\rangle \otimes|n\rangle+\gamma|1\rangle \otimes|n-1\rangle),
$$

with the coefficients $\beta$ and $\gamma$ which are equal

$$
\begin{aligned}
& \beta=\sin \left(\vartheta_{n} / 2\right)=\frac{1}{\sqrt{2}}\left(\frac{\Omega_{n}(\Delta)-\Delta}{\Omega_{n}(\Delta)}\right)^{1 / 2}, \\
& \gamma=\cos \left(\vartheta_{n} / 2\right)=\frac{1}{\sqrt{2}}\left(\frac{\Omega_{n}(\Delta)+\Delta}{\Omega_{n}(\Delta)}\right)^{1 / 2},
\end{aligned}
$$

where $\vartheta_{n}=\arctan \left(\frac{\Omega_{n}(0)}{\Delta}\right)$. These entangled states appear, for some periods of time, during the "revival" and

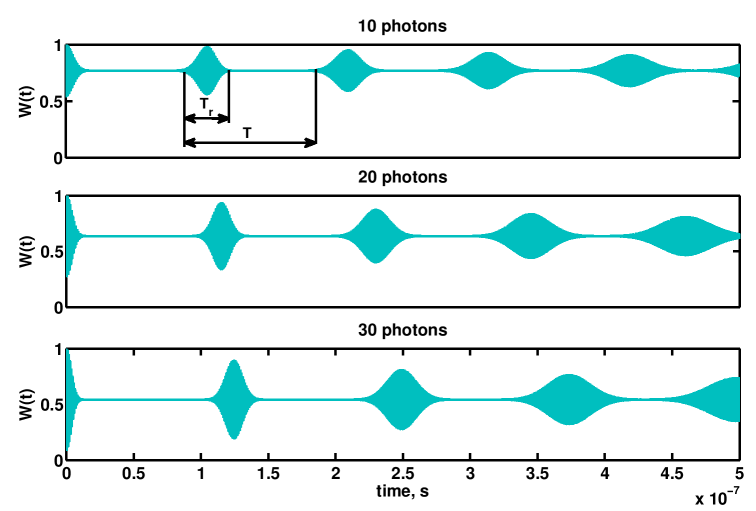

Fig. 4. "Revivals" and "collapses" in population inversion in two-level system interacting with quantized EMfield. 
"collapses" in population inversion in two-level system interacting with quantized EM-field.

For the following values of parameters: $\Delta=\omega-\Omega=$ $5 \times 10^{9} \mathrm{~Hz}, \omega=5 \times 10^{12} \mathrm{~Hz}, g=4.15 \times 10^{8}$ (CGS) we obtain the following "revival" and "collapses", see Fig. 4.

In order to carry out quantum computations we should, at first, adjust the clock frequency of hypothetical quantum computer to the "frequency" $f=1 / T$ of series of revivals and collapses.

\section{Conclusions}

The qubit would arise as a result of quantum entanglement of the symmetric and antisymmetric (with respect to inversion symmetry) states of electrons in DQWstructure. These states arise due to tunneling across the structure, while entanglement could be produced and controlled by means of the source of non-classical light. We examined the possibility to produce quantum entanglement in the framework of JCM and have shown that at least in principle, the entanglement can be achieved due to some interesting phenomena related to JCM, namely series of "revivals" and "collapses" in the interaction of a quantized single-mode EM-field with a two-level system. The other characteristic feature of the entanglement which we propose to get in the ballistic transport in the DQW-structure is that in accordance with our calculations, it can be achieved at relatively high temperature of about $80 \mathrm{~K}$. To our mind, the second advantage of the proposed approach is that one could construct the quantum register by means of single DQW, since the number of quantum levels and hence, the number of symmetric and antisymmetric states and in the consequence, the number of qubits depends only on the depths of QWs and their geometric characteristics. The initial number of quantum levels in a single QW (when the tunneling does not occur) cannot be made of course too great; it is restricted by the available semiconductor materials and it is very unlikely to be greater than four or five. Nevertheless, in this way one can get a small quantum register by means of only the single DQW-structure.

\section{Acknowledgments}

This work was done due to partial support from Centre for Innovation and Transfer of Natural Sciences and Engineering Knowledge at the Rzeszów University.

\section{References}

[1] A. Bertoni, P. Bordone, R. Brunetti, C. Jacoboni, S. Reggiani, Phys. Rev. Lett. 84, 5912 (2000).

[2] R. Ionicioiu, G. Amaratunga, F. Udrea, Int. J. Mod. Phys. B 15, 125 (2001).

[3] N. Tsukada, , in: Eds. T. Ando, Y. Arakawa, S. Komiyama, H. Nakashima, Mesoscopic Physics and Electronics, Springer, 1998.

[4] P. Meystre, M. Sargent, Elements of Quantum Optics, Springer-Verlag, Berlin 1999.

[5] R.K. Schaevitz, J.E. Roth, Shen Ren, O. Fidaner, D.A.B. Miller, J. Sel. Top. Quantum Electron. 14, 1082 (2008).

[6] P. Viscor, O. Andersen, T. Clausen, P. Ellsmore, L. Jensen, J. Schiotz, ECS Transactions 16, 331 (2008).

[7] Xufeng Wang, S. Agarwal, G. Klimeck, D. Vasileska, M. Luisier, J.M.D. Sellier, Piece-Wise Constant Potential Barriers Tool, 2014.

[8] J.H. Davies, The Physics of Low-Dimensional Semiconductors, Cambridge Univ. Press, 2005.

[9] J. Eberly, N. Narozhny, J. Sanchez-Mondragon, Phys. Rev. Lett. 44, 1323 (1980).

[10] N. Narozhny, J. Sanchez-Mondragon, J. Eberly, Phys. Rev. A 23, 236 (1981). 